\title{
Propiedades psicométricas del Inventario de Estilos de Resolución de Conflictos en jóvenes mexicanos
}

\author{
José Luis Rojas', Luz Anyela Morales ${ }^{1}$, Joel Juarros-Basterretxea², Juan Bautista \\ Herrero $^{2}$ y Francisco Javier Rodríguez-Díaz ${ }^{2 *}$
}

'Benemérita Universidad Autónoma de Puebla

2 Universidad de Oviedo

- Recibido: 20 - 01 - 2018 . Aceptado: 08 - 10 - 2018 . Avance online: $21-11-2019$

RESUMEN: La resolución de conflictos tiene vinculación con la satisfacción en la relación de pareja, el bienestar subjetivo o la violencia. El objetivo de este trabajo es analizar las propiedades psicométricas del Inventario de Estilos de Resolución de Conflictos (CSRI; Kurdek, 1994), en el contexto mexicano. La muestra estuvo formada por 1,161 participantes con edades comprendidas entre los 14 y 31 años $(M=20.99$, DT=2.12). El análisis estadístico se realizó a través del programa FACTOR 10.3.01 XP y EQS 6.3. El modelo resultante propone dos factores: Solución positiva de conflictos e Implicación en el conflicto, obteniendo valores de fiabilidad altos en las subescalas (autoinforme entre .823 y .852; y evaluación de la pareja entre .814 y .892) que explican un importante porcentaje de varianza (el autoinformante, por los factores 1 y 2 es de $47.70 \%, 27.67 \%$ y $20.30 \%$, respectivamente para cada uno, y en el caso de la pareja $49.16 \%, 31.39 \%$ y $17.77 \%$, respectivamente para cada uno de los factores) y confirma una relación negativa entre los dos factores. En conclusión, se trata de un instrumento breve y útil para evaluar las estrategias de resolución de conflictos en parejas para el contexto mexicano.

PALABRAS CLAVE: Resolución de conflictos, Propiedades Psicométricas, Pareja, Joven, Mexicano.

\section{Psychometric properties of the Conflict Resolution Styles Inventory in young Mexicans}

ABSTRACT: Conflict resolution is linked to satisfaction in the relationships, subjective well-being or violence. The objective of this work is to analyze the psychometric properties of the Conflict Resolution Styles Inventory (CSRI; Kurdek, 1994), in the Mexican context. The sample composed of 1161 participants aged between 14 and 31 years $(M=20.99, S D=2.12)$. Statistical analysis was performed through FACTOR 10.3.01 XP and the EQS 6.3. The resulting model proposes two factors: Positive solution of conflicts and implication in the conflict, obtaining high reliability values in the subscales (self-report between .823 and .852, and evaluation of the couple between .814 and .892) that explain a significant percentage of variance (the self-information, by factors 1 and 2 is $47.70 \%, 27.67 \%$ and $20.30 \%$, respectively for each, and in the case of the couple $49.16 \%, 31.39 \%$ and $17.77 \%$, respectively for each of the factors) and the confirmation of a negative relation between the two factors. In conclusion it is a brief and useful tool for evaluating conflict resolution strategies in pairs for the Mexican context.

KEYWORDS: Conflict resolution, Psychometric Properties, Partner, Young, Mexican.

Se ha venido documentando que el conflicto en las relaciones interpersonales de los y las jóvenes tiene implicaciones importantes para la salud psicológica y física, así como para el bienestar general (Alarcón, Pérez-Luco, Wenger, Salvo, y Chesta, 2018; Gallegos-

\footnotetext{
*Correspondencia: Francisco Javier Rodríguez-Díaz.

Universidad de Oviedo.

Código Postal: 33003, Facultad de Psicología. Plaza Feijóo, s/n.

Oviedo, España.

E-mail: gallego@uniovi.es
}

(c) 2018 Sociedad Universitaria de Investigación en Psicología y Salud. Publicado por Consejo General de Colegios Oficiales de Psicólogos, España. Este es un artículo Open Access bajo la CC BY-NC-ND licencia (http://creativecommons.org/licencias/by-nc-nd/4.0/).
Guajardo, Ruvalcaba-Romero, Castillo-López, y Ayala-Díaz, 2016; Juarros-Basterretxea, Herrero, Fernández-Suárez, Pérez, y RodríguezDíaz, 2018). Además, existe una creciente evidencia de que el conflicto y su gestión, así como la subsiguiente disolución de nuestras relaciones interpersonales, están implicados en los problemas sociales, emocionales y de comportamiento que afectan al bienestar de las personas (González-Villalobos, y Marrero, 2017; Ruvalcaba-Romero, Murrieta-Cummings, y Artega-Velazquez, 2016). Por estas razones, 
el conflicto se entiende como la principal razón para la ruptura de cualquier tipo de relación interpersonal, facilitando ello la comprensión de cómo el conflicto conduce a la aparición de la angustia en las relaciones interpersonales de los jóvenes (Férriz, Sobral, y Gómez-Fraguela, 2018; Siödin, Wallinius, Billstedt, Hofvander, y Nilsson, 2017). De hecho, la investigación empírica indica que ciertas estrategias de resolución de conflictos de los jóvenes, los comportamientos interpersonales utilizados para abordarlos, son indicadores sobresalientes de la satisfacción en sus relaciones interpersonales (Moyano, Monge, y Sierra, 2017; Zafirakis, 2015).

Se desprende claramente de la literatura sobre los estilos de resolución de conflictos de los adolescentes una vinculación, entre otras cosas, con la satisfacción en la relación, el bienestar subjetivo y la violencia. Por ejemplo, un estilo de implicación directa en el conflicto se asocia con el detrimento de la satisfacción en la relación interpersonal (Kurdek, 1994); el empleo de estrategias positivas de resolución lleva a la reducción de la violencia ejercida dentro de la díada (Benítez y Muñoz, 2014); las estrategias de implicación directa y las de retirada producen un menor bienestar subjetivo (Siffert y Schwarz, 2010). Además, se ha encontrado evidencia de que los jóvenes agresores de género tienden a emplear con mayor frecuencia estrategias de afrontamiento de retirada (Novo, Herbón, y Amado, 2016).

El interés en explicar la satisfacción y la estabilidad en la trayectoria de las relaciones interpersonales ha sido punto central en diferentes estudios de parejas (Schoebi, Karney, y Bradbury, 2012); a la vez, estudios previos han demostrado la eficacia de las intervenciones, a través de programas de entrenamiento de desarrollo de habilidades de resolución de problemas, para reducir los conflictos y aumentar la satisfacción en las relaciones interpersonales de los ióvenes (por ejemplo, Askari, Mohd, Aishah, y Baba, 2013; Perrone-McGovern et al., 2014; Robertson, Fish, Olmstead, y Fincham, 2015). Se ha constatado, incluso, que es posible la mejora de las estrategias de afrontamiento en agresores de género a partir de un entrenamiento reeducativo específico (Arce, Fariña, y Novo, 2014).

Por lo anterior, la evaluación de las habilidades de resolución de conflictos resulta crucial entre nuestros jóvenes (Bringas-Molleda et al., 2017; Martín-Fernández, Gracia, Marco, Vargas, Santirso, y Lila, 2018); por ejemplo, para conocer diferentes estilos y sus efectos en las relaciones de pareja, para establecer líneas de base en intervenciones o terapias, evaluar la efectividad de programas dirigidos a su mejoramiento asociado con variables como la satisfacción, el bienestar o la violencia, para comparar diferentes grupos poblacionales (por género, relaciones heterosexuales, homosexuales, etc.) En ese sentido la evaluación de los estilos de resolución de conflictos en parejas jóvenes ha llevado a diseñar diversos instrumentos (Garaigordobil, Machinbarrena, y Maganto, 2016), muchos de los cuales confluyen en la referencia a la violencia en la pareja.

Kurdek (1994), en esta línea, creó un inventario específico de Resolución de Conflictos (CRSI). Este ofrece como base cinco temas recurrentes en la investigación sobre las relaciones íntimas heterosexuales: 1) las mujeres se definen a sí mismas en términos de sus relaciones; 2) los hombres dan mayor importancia a la autonomía que las mujeres; 3 ) la contribución de hombres y mujeres a la relación no es equitativa; 4) hombres y mujeres tienen dificultad para resolver de manera constructiva los conflictos de su relación; y 5) las barreras institucionales son necesarias para estabilizar las relaciones interpersonales afectivas. EI CRSI en su versión original, constituido como autoinforme para evaluación de la resolución del conflicto en la pareja, va a identificar cuatro estrategias: 1) solución positiva de conflictos, que implica compromiso y negociación (se centra en el problema y lo resuelve constructivamente, tratando de encontrar alternativas aceptables para ambos); 2) implicación directa en el conflicto, caracterizada por ataques personales y pérdida de control (explota y ataca al compañero, con insultos e insinuaciones, dice cosas que él o ella no quieren decir); 3) evitación o retirada, en tanto que no se aborda un tema problemático (pone barreras a la comunicación, se cierra, se niega a hablar y se distancia físicamente de la pareja, el o ella está en silencio durante largos períodos de tiempo, habla con otras personas que están fuera de la conversación, es distante y actúa como si él o ella no estuviese interesado en la conversación); y 4) conformismo, cuando la persona cede ante el conflicto y no defiende 
su punto de vista (actitudes de una persona que no se siente a gusto con él mismo cuando su posición es evidente en una situación de conflicto; se siente demasiado sumiso, no defiende su posición, y siente poca inclinación a expresarlo en el evento). Estas estrategias van a ofrecer una fiabilidad optima, tanto para hombres (Coeficientes Alfa respectivamente de .76, .76, .82 y .71) como para mujeres (Coeficientes Alfa de.71, .75, .81 y .75),así como evidencia de validez concurrente y predictiva de criterio relacionado con correlaciones moderadas (de -20 a .42) entre estilos de resolución de conflictos y construcciones disimilares, tales como la satisfacción conyugal (Kurdek, 1994, 1998).

Kurdek (1998), en estudios posteriores, planteará solo tres dimensiones (resolución positiva de conflictos, implicación en el conflicto y evitación). Estas dimensiones evaluarían una variable que llamó "solución constructiva de problemas", sin realizar ninguna evaluación de ajuste del modelo a estos factores. En esta línea, Bonache, González-Méndez, y Krahé (2016, 2017) confirmaron recientemente este modelo mediante análisis factorial confirmatorio del CRSI original de cuatro dimensiones. Los resultados indican que el modelo de cuatro factores (Kurdek, 1994) no muestraun buen ajuste; resulta una estructura de tres factores: estrategias positivas, implicación en el conflicto y evitación, donde el ítem 12 ("no defender la propia opinión") se incluye en Retirada. La fiabilidad obtenida para las subescalas resulta optima: alfa de Cronbach de .76 (para el autoinforme) y de .73 (para la pareja).

En México existen algunas escalas que evalúan tópicos afines a los estilos de resolución de conflictos en parejas, pero no de jóvenes. Son de destacar: la Escala de conflicto familiar para latinos (Cárdenas, Mata, Vite, y Flores, 2002), donde se evalúan antecedentes y situaciones bajo los cuales se precipita el comportamiento violento y las estrategias utilizadas para afrontar los conflictos familiares; la Escala de estilos conductuales (Rivera, 2000), que mide el modo particular en el que una persona tiende a comportarse con su pareja; la Escala de Estilos de negociación (Rivera, 2000), que evalúa el modo particular en el que una persona llega a un acuerdo con su pareja sobre un asunto dado; y la Escala de estrategias de poder (Rivera-Aragón, Díaz-Loving, y Manrique, 2000), donde se explora autoritarismo, afecto positivo y negativo, equidad-reciprocidad, imposiciónmanipulación, descalificación, coerción, chantaje y dominio. Si bien éstas son escalas que pueden ser muy útiles en el estudio de las relaciones interpersonales de los jóvenes, tales como las afectivas, aún es escaso el diseño y validación de instrumentos para evaluar específicamente en diadas las estrategias de resolución de problemas. Son excepción, en ello, los esfuerzos realizados por Mora, Natera, Tiburcio, y Juárez (2008), Hokoda et al.(2006) y Antonio y Hokoda (2009), aunque sus instrumentos están más orientados a la evaluación de violencia en la pareja,que a los estilos de resolución de conflictos en relaciones relaciones interpersonales, y menos aún de parejas de jóvenes.

Por lo anterior, el estado actual de conocimientos y de evaluación de los estilos de resolución de problemas en relaciones interpersonales en parejas latinoamericanas, y en concreto de México, refiere la necesidad de proponer la adaptación de un modelo que se ajuste a este contexto. Ello nos lleva a plantear como objetivo del estudio el analizar las propiedades psicométricas del CRSI, adaptado al castellano por Bonache, Ramírez-Santana,y González (2016) y desarrollado originalmente por Kurdek (1994), en el contexto de México.

\section{MÉTODO}

\section{-MUESTRA}

El tipo de selección de los participantes fue no probabilístico e intencionado. Así, la muestra estuvo formada por un total de 1,161 sujetos, con edades comprendidas entre los 14 y los 31 años $(M=20.99$; $D T=2.12)$. El $60.38 \%(n=701)$ eran mujeres y el $39.62 \% \quad(n=460)$ restante hombres. Las participantes refirieron una edad media de 20.85 años $(D T=2.00)$, con un mínimo de 14 años y un máximo de 31 ; por su parte, los varones tuvieron una edad media de 21.21 años $(D T=2.27)$, oscilando entre los 17 y los 31 años. El $60.55 \%(n=703)$ de los participantes tenía pareja en el momento de la investigación, mientras que el $39.45 \%$ ( $n=455)$ no; concretamente, el $66.07 \%$ $(n=442)$ de las mujeres y el $56.86 \%(n=261)$ de los hombres que participaron tenían pareja en el momento de realizar el estudio. 


\section{- INSTRUMENTOS}

El Inventario de Estilos de Resolución de Conflictos (CRSI; Kurdek, 1994) consta de 16 ítems, que fueron inicialmente agrupados en cuatro estilos (Positivo, Implicación conflictiva, Retirada y Conformidad). Se aplica a los participantes la versión de tres dimensiones (estrategias positivas, implicación en el conflicto y evitación) propuesta por Bonache, RamírezSantana et al. (2016), constituido por 13 ítems, resultante del Inventario de Resolución de Conflictos creado por Kurdek (1994), adaptado a la población adolescente de España.Los participantes indicaran la frecuencia con la que ellos mismos (CRSI-Yo) y sus parejas (CRSI-Pareja) empleaban las 13 estrategias. Las respuestas de las subescalas van de 1 (nunca) a 5 (siempre). Los coeficientes de fiabilidad son adecuados en la adaptación a adolescentes españoles, alcanzando alfas de Cronbach de .76 (CRSI-Yo) y .73 (CRSI-Pareja), sin observar ninguna mejora en el alfa al excluir algún ítem; otros investigadores también han encontrado evidencia de una buena consistencia interna del CRSI en muestra de adolescentes, alcanzando coeficientes alfa de Cronbach entre .77 y .84 , respectivamente $(\mathrm{Ha}$, Overbeek, Cillessen, y Engels, 2012).

\section{-PROCEDIMIENTO Y ANÁLISIS DE DATOS}

En cuanto a la recolección de datos, en primer lugar, se contactó con la directiva de diversas facultades y se obtuvo la autorización para desarrollar el estudio, explicando la naturaleza y los objetivos del mismo durante la aplicación de las pruebas. La recogida de datos tuvo lugar en las aulas de cómputo de las facultades, durante horario escolar, siendo la duración de la aplicación de aproximadamente veinte minutos, explicando las instrucciones correspondientes y la naturaleza del estudio. Los aspectos éticos han sido seguidos de acuerdo con las normas sugeridas por la Sociedad Mexicana de Psicología (2007), así como aquellas propias de la investigación por medios virtuales (Hoerger y Currell, 2012). De esta manera, se ha integrado un ítem específico de consentimiento informado, que en caso de no obtener respuesta afirmativa, anularía la participación en el estudio.

El análisis estadístico de los datos se realizó a través del programa FACTOR 10.3.01 XP y el EQS 6.3. La fiabilidad marginal, a su vez, se ha estimado a partir de las estimaciones EAP (Ferrando y Lorenzo-Seva, 2016) y el estudio de la normalidad de las puntuaciones mediante la prueba de Kolmogorov-Smirnov (K-S).

El análisis factorial confirmatorio (AFC) se ha realizado para comprobar el ajuste del modelo propuesto por Bonache, Ramírez-Santana et al. (2016) a la muestra, empleando para ello la totalidad de la misma. Posteriormente, se ha procedido a dividir la muestra de forma aleatoria en tres grupos homogéneos compuestos por 395,381 y 385 sujetos respectivamente. A tenor de los resultados obtenidos se ha realizado un análisis factorial exploratorio (AFE) con la primera de las submuestras $(n=395)$, siendo el ajuste establecido mediante el Adjusted Goodness of Fit Indez (AGFI) y la Root Mean-Square of Residuals (RMSR). La pertinencia de someter los datos a análisis factorial se estima mediante el índice de Bartlett y el test de Kaiser-Meyer-Olkin, siendo el método de extracción el de mínimos cuadrados no ponderados y el método de rotación el promin. Las submuestras $2(n=381)$ y $3(n=385)$ se han empleado para llevar a cabo un análisis factorial confirmatorio (AFC) utilizando el método de validación cruzada, con el fin de tener en cuenta las correlaciones entre los errores en la modelación de la primera muestra para mantenerlo en la segunda y encontrar el ajuste más adecuado; el método de extracción de factores utilizado fue de máxima verosimilitud robusta, sobre matriz de correlaciones policóricas, siendo el ajuste de los datos al modelo establecido por el $\chi^{2} / \mathrm{g} .1$. de Satorra-Bentler o robusto, el índice de Ajuste Comparativo (CFI) y la Root Mean-Square Error of Approximation (RMSA).

\section{RESULTADOS}

La necesidad de contar con una prueba de evaluación de estilos de resolución de problemas en las las relaciones interpersonales de pareja, para el contexto de México, ha llevado a proponer la adaptación del modelo de Kurdek (1994) ya adaptado por Bonache, Ramírez-Santana et al. (2016) a población joven de España. El análisis factorial confirmatorio realizado constata que el modelo propuesto por Bonache Ramírez-Santana et al. (2016) no tiene un buen ajuste a los datos, ni en lo referente al propio sujeto (self) (modelo A) ni en el relativo a la pareja (modelo B), tal y 
Tabla 1

Nivel de ajuste de los datos al modelo tridimensional para el sujeto (self) (modelo A) y la pareja (modelo B) mediante análisis factorial confirmatorio

\begin{tabular}{|c|c|c|c|c|}
\hline Modelo & $\chi_{S-B}^{2}$ & $d f$ & CFI Robusto & RMSA Robusto \\
\hline A & 257.5384 & 64 & .916 & $.051(.045, .058)$ \\
B & 233.3775 & 64 & .929 & $.048(.041, .054)$ \\
\hline \multicolumn{2}{|l}{ Nota. El modelo A (autoinforme) y el modelo B (pareja) se han calculado de forma independiente. } \\
\hline
\end{tabular}

como muestran los índices robustos presentados en la Tabla 1.

Los resultados obtenidos en el análisis confirman que los factores 1 (conflict engagement/Implicación directa en el conflicto) y 3 (withdrawal/Retirada), cuya relación ya fue puesta de manifiesto en estudios anteriores (Bonache, Ramírez-Santanaet al., 2016), tienen una correlación positiva elevada entre sí: concretamente, es de .85 en el caso del autoinforme y de .90 en la pareja, siendo estadísticamente significativa en ambos casos $(p<.05)$. Además, es posible comprobar que son varias las fuentes de error cuya correlación mejoraría el ajuste del modelo a los datos.

Estos indicios respaldan la posibilidad de que se trate de un único factor. Para confirmarlo se ha llevado a cabo un análisis factorial exploratorio con la submuestra 1, cuyos resultados recomiendan la extracción de dos factores, tanto para la persona autoinformante como para la pareja; el estadístico de Bartlett, $\left(\chi^{2} 66\right)$ $\left.=1180.1, p<.001 ; \chi^{2}(66)=1280.9, p<.001\right)$ y el test de Kaiser-Meyer-Olkin $(.80, .84)$ indican que los datos cumplen las condiciones para ser sometidos a análisis factorial. Los valores de la matriz de rotación (ver Tabla 2) constatan que el ítem 10 ("No defiendes tu propia opinión/no defiende su propia opinión") debe ser excluido del modelo por su baja comunalidad $(<.10)$ en ambos casos:

El porcentaje de varianza explicada, en el caso del auto-informante, por los factores 1 y 2 es del $47.70 \%$ (27.67\% y $20.30 \%$, respectivamente para cada uno de los factores) y del $49.16 \%$ (31.39\% y $17.77 \%$, respectivamente para cada uno de los factores) en el caso de la pareja. A su vez, los resultados del AFE indican que existe una relación negativa entre los factores 1 y 2 , tanto en el caso del auto-informante (-.185) como en el de la pareja (-.331). Los índices de ajuste obtenidos son adecuados, siendo los índices de fiabilidad satisfactorios en ambos casos para los factores $(\alpha \geq .80)$.

A continuación, la Tabla 3 ofrece los resultados del AFC llevado a cabo con las submuestras 2 y 3 . Es el resultado de poner a prueba el ajuste del modelo propuesto en el presente artículo, a partir de los datos obtenidos en el AFE. Los índices robustos del análisis factorial confirmatorio, llevado a cabo para poner a prueba el modelo alternativo mediante el

Tabla 2

Comunalidades de la matriz de rotación del análisis factorial exploratorio con la submuestra 1

\begin{tabular}{|c|c|c|c|}
\hline Ítems & Negativo Self & Negativo Otro & Positivo Self \\
\hline 1 & .506 & .578 & \\
3 & .503 & .416 & \\
4 & .553 & .638 & \\
6 & .689 & .652 & \\
7 & .612 & .735 & \\
\hline
\end{tabular}


Tabla 2 (Continuación)

Comunalidades de la matriz de rotación del análisis factorial exploratorio con la submuestra 1

\begin{tabular}{|c|c|c|c|c|}
\hline Ítems & Negativo Self & Negativo Otro & Positivo Self & Positivo Otro \\
\hline 9 & .500 & .484 & \\
10 & .483 & .559 & \\
11 & .650 & .672 & .534 & \\
13 & & & .784 & .535 \\
2 & & & .758 & .713 \\
5 & & & .646 & .679 \\
8 & & & \\
12
\end{tabular}

Tabla 3

Resultados del Análisis Factorial Confirmatorio llevado a cabo con submuestras 2 y 3

\begin{tabular}{|c|c|c|c|c|}
\hline Modelo & $\chi_{\text {S-B }}^{2}$ & $d f$ & CFI Robusto & RMSA Robusto \\
\hline $\mathrm{C} 1$ & 382.6508 & 234 & .955 & $.041(.033, .048)$ \\
$\mathrm{C} 2$ & 400.7453 & 234 & .951 & $.043(.036, .050)$ \\
\hline
\end{tabular}

método de validación cruzada, indican un buen ajuste del modelo a los datos.

El modelo obtenido, tal y como puede verse en la figura 1, lo conforma un total de cuatro factores: dos relativos al auto-informante y otros dos equivalentes a la pareja. Se asume que los factores están relacionados entre sí y que los errores de los ítems equivalentes (p.e. ítem 1A y 1B) están relacionados entre sí.

Los índices de fiabilidad marginal EAP obtenidos para cada uno de los factores del modelo del 'self' $\left(\alpha_{\mathrm{p}}=.888 ; \alpha_{\mathrm{n}}=.860\right)$ y de la pareja $\left(\alpha_{p}=.879 ; \alpha_{n}=.878\right)$ son altos.

\section{DISCUSIÓN}

El estudio de las estrategias de resolución de conflictos interpersonales en nuestras relaciones presenta importantes limitaciones en el contexto latinoamericano, puesto que los pocos instrumentos disponibles están enfocados en la evaluación de la violencia en la pareja, y principalmente de población adulta (Antonio y Hokoda, 2009; Delatorre, Scheeren, y Wagner, 2017; Hokoda et al.,2006; Mora et al., 2008). Por ello, el objetivo de esta investigación ha sido analizar las propiedades psicométricas del CRSI, creado por Kurdek (1994) y adaptado al castellano por Bonache, Ramírez-Santanaet al. (2016). Este instrumento evalúa específicamente las estrategias de resolución de conflictos en parejas, a través de un auto-informe y evaluando a la pareja; dado que es breve puede aplicarse en poco tiempo a grandes muestras, lo que puede considerarse como una contribución práctica al desarrollo de la línea 


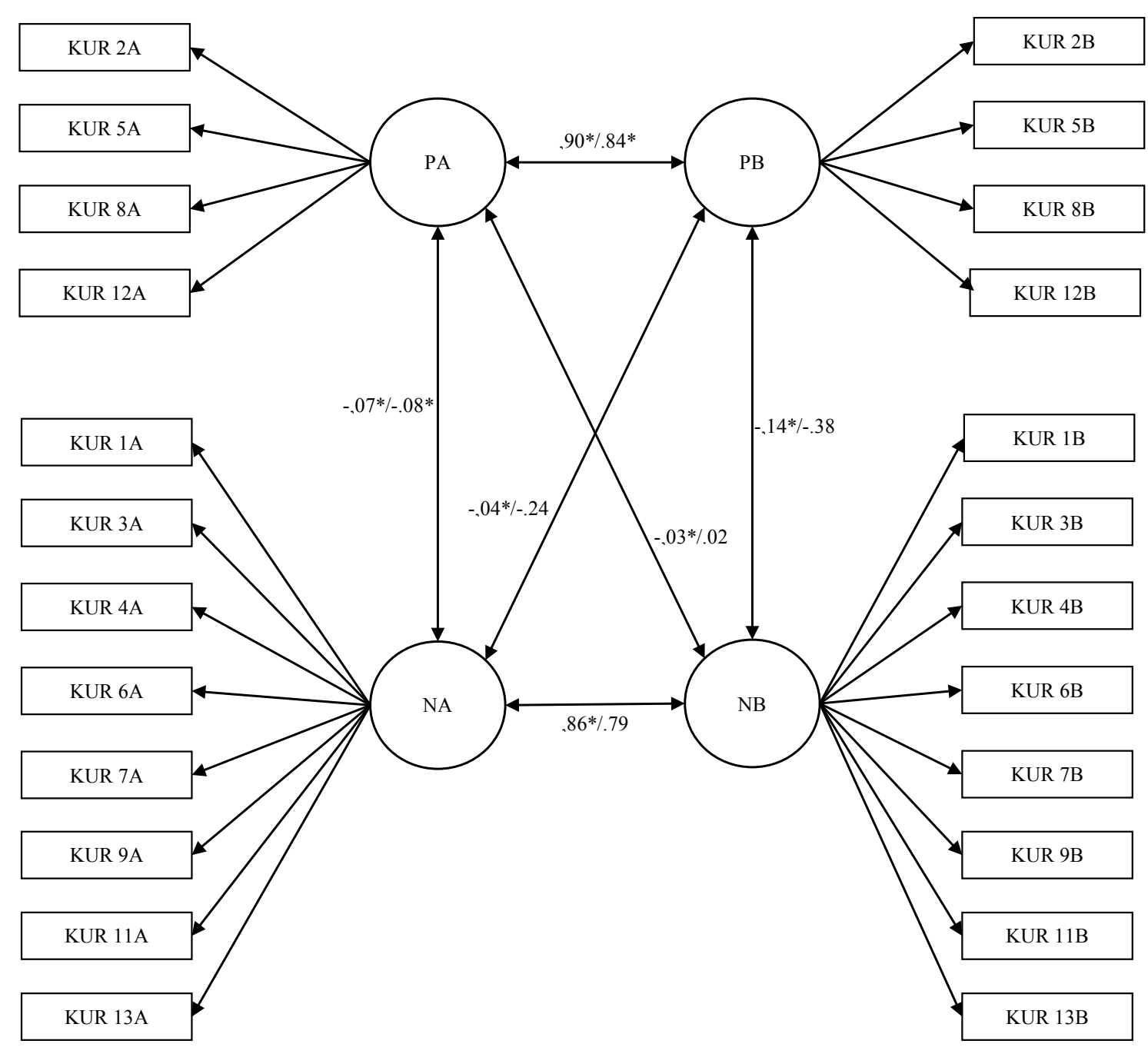

Figura 1. Modelo resultante del estudio (Elaboración propia. PA=Factor Positivo Self; PB=Factor Positivo pareja; $N A=F a c t o r$ Compromiso en conflicto Self; NB=Factor compromiso en conflicto Pareja).

de investigación en el conflicto de relaciones interpersonales en el contexto de México.

Los resultados de este estudio señalan, al menos para el contexto de México, que no se confirma la estructura de tres factores CRSI propuesta por Bonache, Ramírez-Santana et al. (2016) en jóvenes españoles. El modelo resultante de la presente investigación propone dos factores ( $F$ l. Solución positiva de conflictos; y F2. Implicación directa en el conflicto), tanto en el formato de auto-informe como en el de evaluación de la pareja. Además, los índices de fiabilidad obtenidos para cada uno de los factores (en el auto-informe entre .823 y .852; y para la evaluación de la pareja entre .814 y .892) fueron superiores a los informados por Bonache, Ramírez-Santana et al. (2016) de .76 para el auto-informe y de .73 para la pareja.
El modelo de dos factores muestra valores de fiabilidad altos en las subescalas, que explican un importante porcentaje de varianza $(47.7 \%$ en el auto-informe y $49.16 \%$ en la evaluación de la pareja), y la confirmación de una relación negativa entre los dos factores, dado que evalúan tipos diferentes de estrategias: positivas(compromiso y negociación) vs.implicación directa en el conflicto (que contiene ítems del factor original de evitación, además de los propios de la implicación en el conflicto).

Este modelo coincide con estudios previos, como el de Hennigan (2013), que ha encontrado que la mayoría de respuestas en el CRSI se registran en dos de los cuatro factores; su investigación, dado que la mayor frecuencia de respuestas se registró en las estrategias positivas 
y que las respuestas en los otros tres factores fueron escasas, permitiría factores por diadas, encontrando que la diada que agrupaba mayor número de respuestas estaba compuesta por las estrategias de implicación en el conflicto y las de evitación. Estos resultados se confirman en nuestro estudio a través de los ítems que conforman el F2 en nuestro modelo.

La aplicación de la adaptación del CRSI de Bonache, Ramírez-Santana et al. (2016) a parejas adolescentes ofrece correlaciones significativas y positivas entre las medidas de violencia (perpetración y victimización) y las dos estrategias de resolución de conflictos no constructivas (implicación en el conflicto y evitación); el uso de estrategias no constructivas de resolución de conflictos se asoció con un mayor nivel de victimización y perpetración en las relaciones de adolescentes, a la vez que de nuevo los dos factores muestran una relación simultánea, y en la misma dirección, con variables relevantes en la relación interpersonal de pareja (Bonache, González-Méndez, y Krahé, 2017).

La implicación en el conflicto y la evitación parece, pues, suelen combinarse en el patrón de demanda-retirada (Christensen, Eldridge, CattaPreta, Lim, y Santagata, 2006). Ambas estrategias se asocian con el inicio de agresiones. Frente a ello, Bonache, González-Méndez et al. (2016) y Bonache, González-Méndez, y Krahé (2017) sostienen que los factores implicación en el conflicto y evitación se han mostrado como estilos diferentes tanto en el CRSI como en otros instrumentos. Estos estilos corresponden con el patrón demanda/evitación, que ha sido consistentemente relacionado con la baja satisfacción en las relaciones. Es decir, mientras una parte de la pareja discute y demanda cambios, la otra parte los evita con silencio o indiferencia (Christensen et al., 2006; Eldridge, Sevier, Jones, Atkins, y Christensen, 2007).

Los resultados de nuestro modelo ofrecen los ítems originales de los patrones de demanda/ evitación (F2) en un mismo. Ello, en la línea del estudio de López (2015), puede referir y prever relaciones conflictivas en nuestras parejas de jóvenes. Es de mencionarse que el ítem: "No defiendes tu propia opinión" (autoinforme); "No defiende su propia opinión" (evaluación de la pareja), que originalmente estaba en el Factor 4: Conformismo (Kurdek, 1994) y que luego en el modelo trifactorial de Bonache, Ramírez-Santana et al. (2016) fue incluido en el Factor 3: Evitación, en nuestra investigación ha sido excluido del modelo debido a su baja comunalidad. Nuestro resultado, pues, confirma la dificultad de ajuste de este ítem.

Igualmente, es de resaltarse que nuestra investigación ofrece un modelo del CRSI breve, útil y objetivo para evaluar las estrategias de resolución de conflictos en parejas para el contexto mexicano. No debemos olvidar en ello que la muestra de nuestro estudio se ha conformado con estudiantes de preparatoria y universidad, por lo que sus niveles de generalización se ven limitados a este sector de la población; sin embargo, el tamaño muestral empleado para la aplicación ofrece resultados interesantes y el modelo se muestra prometedor para el empleo del CRSI en población mexicana, a la vez que contribuye a la línea de investigación sobre la violencia en las relaciones interpersonales afectivas en pareja de jóvenes.

A la par, los resultados obtenidos constatan una clara discrepancia con los estudios realizados previamente por Kurdek (1994), Han et al. (2007) y Bonache, Ramírez-Santana et al. (2016). Ello podría defenderse y entenderse como producto de diferencias culturales entre las muestras y/o diferencias en otras características más específicas: en el estudio de Bonache et al. (2016) se evaluaron 592 adolescentes (47\% hombres y $53 \%$ mujeres), en nuestra investigación fueron 1161 participantes (60\% mujeres y $40 \%$ hombres) con edades entre los 14 y los 31 años; el estudio de Kurdek (1994) se conforma con una muestra de adultos, 75 parejas homosexuales, 51 parejas lesbianas y 207 parejas heterosexuales. Estas observaciones nos llevan a sugerir la importancia para futuras investigaciones de comprobar el funcionamiento diferencial del modelo en adolescentes y adultos jóvenes y profundizar en cuanto a género, nivel educativo, tipo de parejas, personas con y sin historial delictivo relacionado con violencia en la pareja. A su vez, dado que el instrumento ha sido aplicado de forma individual, sin contar con el control que significa la aplicación simultánea a los miembros de la pareja, las respuestas pueden estar influenciadas por los sesgos de deseabilidad social. Vale la pena considerar esta situación, igualmente, para investigaciones futuras y contemplar en las muestras no solo individuos sino parejas, a la vez que conformar 
su validez de constructo en la predicción tanto del agresor como de la víctima en las relaciones interpersonales afectivas de parejas jóvenes.

Nuestros resultados, de esta manera, posibilitarán el inicio de una línea de investigación para configurar una herramienta potente, que permitirá identificar ventajas y debilidades en el proceso de resolución de conflictos en relaciones afectivas de parejas jóvenes, a la vez que evaluar los resultados de programas de entrenamiento en este tipo de habilidades para nuestros jóvenes y fortalecer las líneas de investigación futura.

Dos son las limitaciones que se pueden atribuir al método, esto es, la varianza atribuible al método de medida y no al constructo medido. Primera, la disimulación; en este caso, deseabilidad social en las repuestas de los participantes (Arce, Fariña, Seijo, y Novo, 2015). Segunda, los instrumentos breves captan menos medida del constructo (validez), especialmente cuando la consistencia interna es elevada sustantada en la eliminación de ítems con menos comunalidad con la escala y, por tanto, con mayor aportación a la medida (Salvador, Arce, Rodríguez-Díaz, y Seijo, 2017).

\section{- Conflicto de intereses}

Los autores declaran no tener ningún conflicto de intereses.

\section{REFERENCIAS}

Alarcón, P. A., Pérez-Luco, R. X., Wenger, L. S., Salvo, S. I., y Chesta. S. A. (2018). Personalidad y gravedad delictiva en adolescentes con conducta antisocial persistente [Personality and offense severity in adolescents with persistent antisocial behavior]. Revista Iberoamericana de Psicología y Salud, 9(1), 58-74. https:// doi.org/10.23923/i.rips.2018.01.015

Antonio, T., y Hokoda, A. (2009). Gender variations in dating violence and positive conflict resolution among Mexican adolescents. Violence and Victims, 24(4), 533-545. https://doi.org/10.1891/0886$\underline{6708.24 .4 .533}$

Arce, R., Fariña, F., y Novo, M. (2014). Competencia cognitiva en penados primarios y reincidentes. Implicación para la reeducación [Cognitive competence among recidivist and non-recidivist prisoners:
Implications for the rehabilitation]. Anales de Psicología, 30(1), 259-266. http://dx.doi. org/10.6018/analesps.30.1.158201

Arce, R., Fariña, F., Seijo, D., y Novo, M. (2015). Assessing impression management with the MMPI-2 in child custody litigation. Assessment, 22(6), 769-777. http://dx.doi. org/10.1177/1073191114558111

Askari, M., Mohd, S., Aishah, S., y Baba, M. (2013). Comparison of the effects of communication and conflict resolution skills training on mental health. International Journal of Psychological Studies, 5(1), 91 . 104. https://doi.org/10.5539/iips.v5n 1 p91

Benítez, J. L., y Muñoz, J. F. (2014). Análisis factorial de las puntuaciones del CADRI en adolescentes universitarios españoles. Universitas Psychologica, 13(1), 175. 186. https://doi.org/10.11144/Javeriana. UPSY13-1.afpc

Bonache, H., González-Méndez, R., y Krahé, B. (2016). Adult attachment styles, destructive conflictresolution, and theexperience ofintimate partner violence. Journal of Interpersonal Violence. Advance online publication. https:// doi.org/10.1177/0886260516640776

Bonache, H., González-Méndez, R., y Krahé, B. (2017).Romantic attachment, conflict resolution styles, and teen dating violence victimization. Journal of Youth and Adolescence, 46(9), 1905-1917. https://doi. org/10.1007/s10964-017-0635-2

Bonache, H., Ramírez-Santana, G., y González, R. (2016). Conflict resolution styles and teen dating violence. International Journal of Clinical and Health Psychology, 16(3), 276-286. https://doi.org/10.1016/i. iichp.2016.03.003

Bringas-Molleda, C., Estrada-Pineda, C., Suárez-Álvarez, J., Torres, A., Rodríguez-Díaz, F.J., García-Cueto, E., y Rodríguez-Franco, L. (2017). Actitud sexista y trascendente durante el noviazgo entre universitarios latinoamericanos [Sexist and transcendent attitude during courtship between Latin American university students]. Revista Iberoamericana de Psicología y Salud, 8(1), 44-55. https://doi. org/10.23923/i.rips.2017.08.005 
Cárdenas, G., Mata, A., Vite, A., y Flores, E. (2002). Validación y estandarización de la Escala de Conflicto Familiar para latinos. Enseñanza e Investigación en Psicología, 7(2), 281-294.

Christensen, A., Eldridge, K.A., Catta-Preta, A.B., Lim, V.R., y Santagata, R. (2006). Cross-cultural consistency of the demandwithdraw interaction pattern in couples. Journal of Marriage and Family, 68, 1029. 1044. $\quad$ https://doi.org/10.1111/i.17413737.2006.00311.x

Delatorre, M. Z., Scheeren, P., y Wagner, A. (2017). Conflito conjugal: Evidências de validade de uma Escala De Resolução De Conflitos em Casais do sul do Brasil [Marital conflict: Evidences of validity of a Conflict Resolution Scale in Couples in southern Brazil]. Avances en Psicología Latinoamericana, 35(1), 79-94. https://doi.org/10.12804/revistas. urosario.edu.co/apl/a.3742

Eldridge, K. A., Sevier, M., Jones, J., Atkins, D. D., y Christensen, A. (2007). Demandwithdraw communication in severely distressed,moderately distressed, and nondistressed couples: Rigidity and polarity during relationship and personal problem discussions. Journal of Family Psychology, 21, 218-226. https://doi.org/10.1037/08933200.21.2.218

Ferrando, P. J., y Lorenzo-Seva, U. (2016). A note on improving EAP trait estimation in oblique factor-analytic and item response theory models. Psicológica, 37, 235-247. Recuperado de https://www.uv.es/revispsi/ articulos2.16/7Ferrando.pdf

Férriz, L., Sobral, J., y Gómez-Fraguela, J. A. (2018). Empatía y delincuencia juvenil: Un meta-análisis sobre la relación [Empathy and juvenile delinquency: A meta-analytic review]. Revista Iberoamericana de Psicología y Salud, 9(1), 1-16. https://doi.org/10.23923/i. rips.2018.01.011

Gallegos-Guajardo, J., Ruvalcaba-Romero, N.A., Castillo-López, J., y Ayala-Díaz, P.C. (2016). Funcionamiento familiar y su relación con la exposición a la violencia en adolescentes mexicanos [Relationship between family functioning and exposure to violence among Mexican adolescents]. Acción Psicológica, 13(2), 69-78. http://dx.doi.org/10.5944/ ap. 13.2.17810

Garaigordobil, M., Machimbarrena, J. M., y Maganto, C. (2016). Adaptación española de un instrumento para evaluar la resolución de conflictos (Conflictalk): Datos psicométricos de fiabilidad y validez [Spanish adaptation of an instrument to assess conflict resolution (Conflictalk): Psychometric data of reliability and validity]. Revista de Psicología Clínica con Niños y Adolescentes, 3(2), 59-67. Recuperado de http://www.revistapcna.com/ sites/default/files/16-23.pdf

González-Villalobos, J. Á., y Marrero, R. J. (2017). Sociodemographic and personal factors determining subjective and psychological well-being in the Mexican population. Suma Psicológica, 24(1), 59-66. https://doi. org/10.1016/i.sumpsi.2017.01.002

Ha, T., Overbeek, G., Cillessen, A. H. N., y Engels, R. C. M. E. (2012). A longitudinal study of the associations among adolescent conflict resolution styles, depressive symptoms, and romantic relationship longevity. Journal of Adolescence, 35, 1247-1254. https://doi. org/10.1016/i.adolescence.2012.04.009

Hennigan, R. (2013). Successful methods and styles of conflict resolution as identified by heterosexuals, gays, and lesgians in committed relationships a mixed methods study (Tesis Doctoral). Texas Woman's University, Texas.

Hoerger, M., y Currell, C. (2012). Ethical issues in internet research. En S. Knapp, M. Gottlieb, M. Handelsman y L. VandeCreek (Eds.), APA handbook of ethics in psychology (Vol. 2): Practice, teaching, and research (pp. 385-400). Washington, D.C.: American Psychological Association. https://doi. org/10.1037/13272-018

Hokoda, A., Ramos-Lira, L., Celaya, P., Vilhaver, K., Angeles, M., Ruíz, S., Malcarne, V., y Mora, M. (2006). Reliability of translated measures assessing dating violence among Mexican adolescents. Violence and Victims, 21, 117-127. https://doi.org/10.1891/0886$\underline{6708.21 .1 .117}$ 
Juarros-Basterretxea, J., Herrero, J., FernándezSuárez, A., Pérez, B., y Rodríguez-Díaz, F. J. (2018). Are generalist batterers different from generally extra-family violent men? A study among imprisoned male violent offenders. European Journal of Psychology Applied to Legal Context, 10, 8-14. https://doi. org/10.5093/ejpalc2018a 1

Kurdek, L.A. (1994). Conflict resolution styles in gay, lesbian, heterosexual non parents, and heterosexual parent couples. Journal of Marriage and the Family, 56, 705-722. https://doi.org/10.2307/352880

Kurdek, L.A. (1998). Relationship outcomes and their predictors: Longitudinal evidence from heterosexual married, gay cohabiting, and lesbian cohabiting couples. Journal of Marriage and Family, 60, 553-568. https:// doi.org/10.2307/353528

Martín-Fernández, M., Gracia, E., Marco, M., Vargas, V., Santirso, F. A., y Lila, M. (2018). Measuring acceptability of intimate partner violence against women: Development and validation of the A-IPVAW scale. European Journal of Psychology Applied to Legal Context, 10, 26-34. https://doi.org/10.5093/ ejpalc2018a3

Mora, J., Natera, G., Tiburcio, M., y Juárez, F. (2008). Propiedades psicométricas de la Escala de Tácticas de Conflicto (CTS2) en mujeres mexicanas [Psychometric properties of the Conflict Tactics Scale (CTS2) in Mexican women]. Revista Mexicana de Psicología, 25(1), 107-117. Recuperado de http://www. redalyc.org/articulo.oa? id $=243016300008$

Moyano, N.,Monge, F.S., y Sierra, J.C. (2017). Predictors of sexual aggression in adolescents: Gender dominance vs. rape supportive attitudes European Journal of Psychology Applied to Legal Context 9, 25-31. https:// doi.org/10.1016/i.ejpal.2016.06.001 1889-1861

Novo, M., Herbón, J., y Amado, B.J. (2016). Género y Victimización: Efectos en la evaluación de la violencia Psicológica sutil y manifiesta, apego adulto y tácticas de resolución de conflictos. Revista
Iberoamericana de Psicología y Salud, 7(2), 89-97. https://doi.org/10.1016/i. rips.2016.05.002

Perrone-McGovern, K.M., Oliveira-Silva, P., Simon-Dack, S., Lefdahl-Davis, E., Adams, D., McConnell, J., ... Gonçalves, O. F (2014). Effects of empathy and conflict resolution strategies on psychophysiological arousal and satisfaction in romantic relationships. Applied Psychophysiology and Biofeedback, 39, 1925. https://doi.org/10.1007/s10484-0139237-2

Rivera, S. (2000). Conceptualización, medición y correlatos de podery pareja: una aproximación etnopsicológica (Tesis Doctoral). Universidad Nacional Autónoma de México, México D.F., México.

Rivera-Aragón, S., Díaz-Loving, R., y Manrique, L. (2000). Construcción y validación de la escala de estrategias de poder. EnR. DíazLoving, S. Rivera Aragón, el. Reyes Lagunes (Eds.), La psicología social en México (Vol. VIII,pp. 88-94). México, D.F.: AMEPSO.

Robertson, P., Fish, J. N., Olmstead, S. B., y Fincham, F. D. (2015). College adjustment, relationship satisfaction, and conflict management: A cross-lag assessment of developmental "spillover". Emerging Adulthood, 3(4), 244-254. https://doi. org/10.1111/iftr. 12052

Ruvalcaba-Romero, N.A., Murrieta-Cummings, P.,y Arteaga-Velázquez, A. (2016). Competencias socioemocionales y percepción de la comunidad ante la conducta agresiva en adolescentes que viven en entornos de riesgo. Acción Psicológica, 13(2), 79-88. https://doi. org/10.5944/ap.13.2.17816

Salvador, B., Arce, R., Rodríguez-Díaz, F. J., y Seijo, D. (2017). Evaluación psicométrica de la psicopatía: Una revisión metaanalítica [Psychometric assessment of psychopathy: A meta-analytical review]. Revista Latinoamericana de Psicología, 49(1), 36-47. http://dx.doi.org/10.1016/i. rlp.2015.09.015

Schoebi, D., Karney, B. R., y Bradbury, T. N. (2011). Stability and change in the first 10 
years of marriage: does commitment confer benefits beyond the effects of satisfaction? Journal of Personality and Social Psychology, 102(4), 729-7642. https://doi.org/10.1037/ a0026290

Siffert, A., y Schwarz, B. (2010). Spouses' demand and withdrawal during marital conflicto in relation to their subjective well-being. Journal of Social and Personal Relationships, 28(2), 262- 277. https://doi. org/10.1177/0265407510382061

Sociedad Mexicana de Psicología. (2007). Código ético del psicólogo (4a. ed.). México, D.F.: Trillas.
Siödin, A. K., Wallinius, M., Billstedt, E., Hofvander, B., y Nilsson, T. (2017). Dating violence compared to other types of violence: similar offenders but different victims. European Journal of Psychology Applied to Legal Context, 9(2), 83-91. https://doi. org/10.1016/i.eipal.2017.03.001

Zafirakis, E. (2015). Examining interpersonal conflict resolution among persistently antisocial youth - Is this a key mechanism implicated in the persistence of antisocial behaviour? International Journal of Adolescence and Youth, 20, 112-129. https://doi.org/10.108 $\underline{0}$ /02673843.2013.779586 